\title{
Scale, LOINC Axis 5
}

National Cancer Institute

\section{Source}

National Cancer Institute. Scale, LOINC Axis 5. NCI Thesaurus. Code C40569.

The metrological type (formal measurement type) of an observation. For example, some measurements are quantitative (the values for the measurement can be represented as solutions to an algebraic equation), ordinal (the values for the measurement can be placed in rank order), nominal (the values of the observation represent the names of the things that were seen), or narrative (the values of the observation are represented by free text expressions). 